\title{
Questões conceituais e metodológicas quanto ao Reforçamento Não Contingente na intervenção de pessoas com desenvolvimento atípico
}

\author{
Conceptual and methodological issues regarding Non-Contingent Reinforcement in the treatment \\ of people with neurodevelopmental disabilities \\ Aspectos conceptuales y metodológicos relacionados com el Refuerzo No Contingente em el \\ tratamiento de personas com discapacidades del neurodesarrollo
}

Recebido: 02/02/2021 | Revisado: 07/02/2021 | Aceito: 11/02/2021 | Publicado: 17/02/2021

\author{
Claudia Bueno Nogueira \\ ORCID: https://orcid.org/0000-0003-3005-1477 \\ Link Soluções Comportamentais, Brasil \\ E-mail:cnogueir07@gmail.com \\ Andréa Fonseca Farias Lobato \\ ORCID: https://orcid.org/0000-0002-4190-5653 \\ Link Soluções Comportamentais, Brasil \\ E-mail:andreafariasufpa @gmail.com \\ Eugenia Andrea Santos Leão \\ ORCID: https://orcid.org/0000-0001-9109-111X \\ Link Soluções Comportamentais, Brasil \\ E-mail:eugenialeao@gmail.com \\ Romariz da Silva Barros \\ ORCID: https://orcid.org/0000-0002-1306-384X \\ Universidade Federal do Pará, Brasil \\ E-mail: romarizsb@gmail.com
}

\begin{abstract}
Resumo
O reforçamento não contingente (non-contingent reinforcement - NCR) é um procedimento da Análise do Comportamento Aplicada baseado na apresentação de itens ou disponibilização de atividades preferidas de acordo com intervalos de tempo pré-estabelecidos e de forma independente da emissão de respostas-alvo. Apesar de frequentemente ser utilizado com sucesso na redução de comportamentos disruptivos ou problemáticos, não há consenso sobre quais os processos envolvidos e responsáveis pelas mudanças comportamentais observadas. Este artigo teórico-conceitual discute cada um dos possíveis processos relacionados ao NCR e elucidados nos primeiros estudos empíricos sobre o assunto publicados no Journal of Applied Behavior Analysis (JABA) na década de noventa; também considera as contribuições de meta-análises publicadas no JABA na última década e estudos feitos nos últimos três anos para esclarecer os mecanismos pelos quais o NCR suprime respostas. Conclui-se que os efeitos do NCR derivam de uma combinação de fatores que devem ser considerados na construção do "pacote" de intervenção desenhado para cada indivíduo.
\end{abstract}

Palavras-chave: Reforçamento não contingente; NCR; Redução de comportamentos problema; Manipulação de antecedentes.

\begin{abstract}
Non-contingent reinforcement (NCR) is an Applied Behavior Analysis procedure based on the delivery of items or availability of preferred activities within pre-established time intervals and independently of the occurrence of target responses. Although often successfully used to reduce misbehavior, there is no consensus on which processes are involved and responsible for the behavioral changes observed. This conceptual theoretical article discusses each of the processes involved on NCR and elucidated in the first empirical studies on this matter published in the Journal of Applied Behavior Analysis (JABA) in the nineties; it also considers the contributions of studies published in JABA in the last decade in clarifying the mechanisms through which NCR suppresses responses. It is concluded that NCR effects derive from a combination of factors that need to be considered in the construction of the intervention "package" designed for each individual.
\end{abstract}

Keywords: Non-contingent reinforcement; NCR; Problem behavior reduction; Antecedent manipulation.

\section{Resumen}

El refuerzo no contingente, o NCR, es un procedimiento del Análisis de Conducta Aplicado. Este procedimiento se basa en la entrega al individuo de sus objectos preferidos o en la posibilidad de realizar sus actividades preferidas cada 
cierto tiempo, con independencia de sus respuestas. Aunque se utiliza con frecuencia para reducir con éxito los comportamientos disruptivos o problematicos, no existe consenso sobre los procesos involucrados y las causas de los cambios de comportamiento observados. Este artículo teórico-conceptual discute cada uno de los procesos involucrados en el NCR, que han sido dilucidados en los primeros estudios empíricos sobre el tema publicados en el Journal of Applied Behavior Analysis (JABA) en la década de 1990. Este artículo también tiene en cuenta las contribuciones de estudios publicados en JABA en la última década para aclarar los mecanismos por los cuales el NCR suprime las respuestas. Se concluye que los efectos del NCR derivan de una combinación de factores que deben ser considerados en la construcción del "paquete" de intervención diseñado para cada individuo. Palabras clave: Refuerzo no contingente; NCR; Reducción de conductas problemáticas; Manipulación de antecedentes.

\section{Introdução}

O procedimento de análise funcional do comportamento (Iwata, Dorsey, Slifer, Bauman, \& Richman 1982; 1994) permitiu muitos avanços no tratamento de comportamentos inadequados. A partir da identificação da função mantenedora do comportamento problema, é possível elaborar estratégias de intervenção direcionadas especificamente à redução da frequência do comportamento-alvo, aumento de comportamentos alternativos e/ou funcionalmente equivalentes. Dentre os vários procedimentos eficazes na redução de comportamentos problema, o presente artigo destaca o uso do reforçamento não contingente (non-contingent reinforcement - NCR).

O NCR se refere a um conjunto de procedimentos que envolve o acesso ao estímulo preferido (potencialmente reforçador) independentemente da emissão de uma resposta-alvo, geralmente depois de um determinado intervalo de tempo pré-estabelecido (Vollmer, Iwata, Zarcone, Smith, \& Mazaleski, 1993). Ele pode apresentar algumas variações como, por exemplo, quanto à seleção do estímulo preferido (potencialmente reforçador) a ser entregue e ao esquema temporal de apresentação deste estímulo. O reforçador em potencial utilizado durante o procedimento pode ser aquele identificado em uma análise funcional como responsável pela manutenção do comportamento problema em questão, ou outro estímulo identificado como altamente preferido em uma avaliação de preferências, mas não necessariamente funcionalmente equivalente ao reforçador que mantém o comportamento problema. Além disso, o acesso ao item preferido pode ser contínuo ou por intervalos de tempo (fixos ou variáveis). Geralmente, no início de uma intervenção, utiliza-se um esquema mais denso de reforçamento e, aos poucos, se faz um esvanecimento. Por exemplo, quando, a partir de uma análise funcional, avalia-se que os comportamentos inadequados de uma pessoa são mantidos por atenção e ocorrem em média a cada 2 minutos, uma alternativa de intervenção seria oferecer atenção não contingente de forma contínua, ou em um esquema de intervalo de tempo (fixo ou variável), em média a cada 1,5 minutos e gradualmente, ir aumentando o intervalo de tempo entre reforçamentos.

As potenciais vantagens dos procedimentos de NCR sobre outros tratamentos, como o Reforçamento Diferencial de Comportamentos Alternativos (DRA) ou o Reforçamento Diferencial de Outros Comportamentos (DRO) [também conhecido como Reforçamento Diferencial da Omissão do Comportamento-Alvo] incluem: a facilidade de implementação; a eliminação do estado de privação que pode ocorrer em outros tratamentos, quando o responder não atinge o critério estabelecido para a produção do reforço e, a prevenção de explosões de comportamentos (jorro de respostas) durante a extinção, uma vez que um esquema com intervalos pré-estabelecidos garante que o indivíduo terá acesso frequente aos reforçadores (para revisão, ver Carr, Severtson, \& Lepper, 2009).

Quando o DRA é aplicado, espera-se que, à medida em que o comportamento alternativo contate reforçamento, ele venha a aumentar de frequência e, eventualmente, substitua o comportamento problema. Porém, as respostas alternativas nem sempre acontecem na frequência necessária para concorrer com o esquema de reforçamento existente e responsável pela manutenção dos comportamentos inadequados. Muitas vezes, se faz necessário adicionar o procedimento de extinção ao pacote de intervenção, o que consequentemente reduz as oportunidades de acesso ao reforçador quando o comportamento alternativo ainda está em aprendizado e não ocorre com frequência. Já no caso do DRO, o reforço é liberado a partir de um intervalo de 
tempo sem a emissão do comportamento inadequado. Por conseguinte, tanto no caso do DRA com extinção, como no caso do uso do procedimento de DRO, pode-se criar um estado de privação de reforçamento e se observar um aumento temporário na frequência, intensidade, duração e variação na ocorrência de comportamentos inadequados; tal fenômeno é conhecido como explosão de extinção e pode dificultar a continuidade da aplicação e adesão ao plano de intervenção. Com o uso do NCR esses problemas são evitados (Catânia, 2005; Cooper, Heron, \& Heward, 2019).

Apesar das vantagens e aparente atratividade do procedimento de NCR, ainda não se sabe muito sobre os mecanismos pelos quais o mesmo suprime o comportamento (ou ao menos qual o mecanismo mais crítico). Ademais, os estudos experimentais relacionando o NCR à redução de comportamentos indesejáveis em pessoas com TEA e outros casos de desenvolvimento atípico no Brasil são, de um modo geral, escassos em periódicos de Análise do Comportamento indexados de referência no país.

\section{Metodologia}

De acordo com Lakatos e Marconi (2017 - ver também Pereira, Shitsuka, Pereira, \& Shitsuka, 2018), toda pesquisa implica em levantamento de dados, embora possa envolver fontes diversificadas. Esses dados podem ser obtidos por documentação direta, no local onde o fenômeno estudado ocorre (como nas pesquisas de campo ou de laboratório); ou indireta, a partir de dados já coletados por outras pessoas. Neste segundo caso, a pesquisa pode ser documental (fonte primária) ou bibliográfica (que tem como fonte trabalhos de pesquisa já elaborados por outros autores). O presente trabalho é um artigo teórico-conceitual que resulta de uma pesquisa bibliográfica sobre o reforçamento não contingente. Não se trata, portanto, de um artigo de revisão sistemática, mas de uma pesquisa bibliográfica que investigou, seguindo critérios especificados a seguir, os diversos aspectos referentes aos mecanismos críticos para a eficácia do NCR na intervenção analítico-comportamental ao TEA, tendo como fonte os artigos já publicados sobre esse tema, selecionados mediante critério. O artigo, adicionalmente, apresenta uma discussão sobre a evolução do conceito de reforçamento não contingente.

A metodologia empregada consistiu inicialmente no levantamento de todos os artigos publicados sobre o tema no Journal of Applied Behavior Analysis (JABA), que é o principal periódico de Análise do Comportamento Aplicada no mundo. A identificação desses artigos se deu pela busca do termo "Non-contingent Reinforcement" nos títulos, resumos e/ou palavraschave dos artigos.

Como critério para definir o ponto de partida do levantamento, adotou-se o marco temporal da década de noventa, logo após a divulgação dos trabalhos de Iwata e colaboradores (1984;1992) sobre a utilização da análise funcional na identificação das variáveis mantenedoras dos comportamentos-problema. Assim, primeiramente são abordados os estudos relevantes sobre o uso de reforço não-contingente na redução de comportamentos inadequados em pessoas com transtornos do desenvolvimento, publicados no JABA na década de noventa. Foi feita uma análise comparativa de questões conceituais e implicações práticas evidenciadas por todos os estudos publicados. Em seguida, para confrontar esses dados com o que há de mais recente, são apresentadas meta-análises publicadas no JABA nos últimos 10 anos, demonstrando o acúmulo de evidências em favor da efetividade do procedimento e artigos empíricos publicados nos últimos anos, ressaltando o atual estado de conhecimento dos processos envolvidos e dos parâmetros para maior eficiência no uso do procedimento.

Com o propósito de organizar os argumentos apresentados, a estrutura do artigo segue essa estratégia metodológica, começando a abordar os artigos publicados na década de noventa e seguindo para a evolução do campo ao longo dos últimos anos. Na medida em que as questões são apresentadas, incluem-se artigos mais recentes que abordam o tópico em discussão para adicionar sua contribuição, portanto a linha cronológica não é rígida. 


\section{Os Processos Envolvidos no NCR}

O NCR tem sido muito utilizado tanto como condição controle em manipulações experimentais como na redução de comportamentos-problema. Mas, como anteriormente observado, apesar da indiscutível utilidade prática do procedimento, não há consenso quanto aos mecanismos subjacentes e responsáveis pela redução do comportamento, quanto à melhor forma de utilizá-lo e até mesmo quanto à adequação do termo reforçamento "não contingente".

O termo "reforçamento", por definição, refere-se a uma relação de contingência. Segundo Catania (1988), reforçamento é o processo através do qual a probabilidade de emissão de uma resposta aumenta quando esta contata uma determinada consequência imediatamente após a sua ocorrência (contingência), seja ela a adição de um estímulo reforçador ou a remoção de um estímulo aversivo. Assim, está implícito na própria definição de "reforçamento" que o mesmo está relacionado ao efeito da consequência contingente a uma resposta. Carr e Severtson (2005) ressaltam que, por essas razões, é incongruente falar em reforçamento não contingente, bem como enfatizam que o reforçamento geralmente é definido a posteriori e funcionalmente. Eventos são classificados como reforçadores, quando se pode observar um aumento na frequência do comportamento que produz tais eventos. Já com o NCR, o objetivo é reduzir e não aumentar o comportamento-alvo. O que pode haver é um reforçamento adventício de outras respostas que não a resposta-alvo.

De acordo com Carr e Severtson (2005) há pelo menos quatro processos envolvidos nos efeitos do NCR e um termo único não seria suficiente para abarcar todos os mecanismos:

- Quando um estímulo ou evento é disponibilizado em alta taxa de maneira não contingente (alta densidade de apresentação do estímulo potencialmente reforçador), há uma alta probabilidade de que ocorra uma alteração na motivação devido à saciação decorrente do acesso frequente ao potencial reforçador; ou seja, o acesso não contingente pode provocar uma alteração na operação motivadora, na forma de um efeito abatedor na probabilidade de emissão da resposta.

- Com NCR, a contingência (que é uma relação de dependência) entre a resposta e o reforçador é eliminada, porque o reforçador passa a ser entregue em um esquema independente da emissão da resposta. A quebra na "contingência" (resposta-reforçador) pode fazer com que o comportamento entre em extinção.

- $\quad$ O NCR pode, principalmente quando o estímulo potencialmente reforçador está sendo apresentado em um esquema denso, reforçar outras respostas de forma adventícia que, por sua vez, podem temporariamente substituir respostas inadequadas ao contatarem uma maior densidade de reforçamento.

- A apresentação não contingente de um evento reforçador com alta densidade pode reduzir a emissão de respostas inadequadas por uma simples questão econômica. Os fenômenos: matching law (Reed \& Kaplan, 2011) e contraste comportamental (McSweeney \& Norman, 1979) explicam como o organismo aloca respostas para o esquema que oferece uma taxa maior de reforçamento e como alterações na taxa de reforçamento em um determinado esquema podem alterar o responder na direção oposta em outro contexto no qual o esquema de reforçamento permanece inalterado.

Carr e Severtson (2005) argumentam que, mesmo reconhecendo as limitações do termo "reforçamento não contingente", as alternativas propostas ao uso do mesmo, tais como: "reforçamento independente da resposta" e "fuga não contingente" (Poling \& Normand, 1999; Vollmer, 1999) não tiveram adesão da comunidade científica. Os autores concluem que seria melhor manter-se o uso do termo devido a sua maturidade e falta de um nome alternativo mais aceitável. 


\section{Análise dos Estudos sobre NCR Publicados no JABA na Década de 90}

A eficácia do NCR e do DRO no tratamento de comportamentos autolesivos mantidos por reforçamento social positivo foi testada em uma análise comparativa (Vollmer, Iwata, Zarcone, Smith \& Mazaleski, 1993). Três mulheres com déficit cognitivo severo participaram deste estudo. Após a linha de base, as participantes foram expostas a duas condições de tratamento: DRO e NCR. Durante a condição de DRO, o experimentador forneceu atenção de acordo com um esquema de DRO com "reseting", no qual o intervalo de tempo era reiniciado caso o indivíduo emitisse o comportamento alvo durante o intervalo programado; o objetivo era estabelecer um intervalo de 5 minutos de DRO enquanto era mantido um nível baixo de comportamento autolesivo. Durante a condição de NCR, a atenção era fornecida em um esquema de tempo fixo (FT- Fixed Time), no qual o comportamento dos participantes não influenciava na frequência de acesso ao reforçador (idem 1993). Para duas participantes, os efeitos dos tratamentos foram comparados em um delineamento de elementos múltiplos intrassujeito e de linha de base múltipla entre participantes. Um pesquisador foi associado a cada condição, o que foi uma precaução importante para facilitar a discriminação entre as duas condições. Para a terceira participante, os tratamentos foram comparados usando um delineamento com reversão (ibidem, 1993). Os resultados deste estudo demonstraram que o NCR foi tão eficaz quanto o DRO em reduzir comportamentos autolesivos, comprovando a viabilidade do NCR como procedimento de tratamento quando a função comportamental foi identificada.

Ao final desse estudo, Vollmer et al. (1993) levantaram uma série de questões: é necessário iniciar a condição de NCR com atenção contínua? É necessário utilizar esvanecimentos? As apresentações intermitentes de atenção poderiam ser responsáveis pela redução dos comportamentos autolesivos? Os efeitos do NCR seriam resultado de extinção ou seriam resultado de saciação? O reforço adventício poderia ser problemático na condição de NCR? Esses questionamentos inspiraram muitos estudos subsequentes.

Hagopian, Fisher e Legacy (1994) estenderam as descobertas de Vollmer et al. (1993), comparando esquemas densos e mais espaçados de NCR para determinar se era necessário usar esse tipo de esquema no início do tratamento ou se o uso de um esquema mais pobre desde o início poderia produzir resultados semelhantes. A hipótese dos autores era a de que um esquema denso poderia afetar a operação estabelecedora e diminuir a eficácia do reforço, gerando um efeito abatedor na probabilidade de emissão da resposta. Ou seja, quando a atenção fosse fornecida continuamente, não seria necessária a emissão do comportamento alvo.

As participantes desse estudo eram quadrigêmeas de cinco anos de idade, diagnosticadas com déficit intelectual e transtorno global do desenvolvimento, e que apresentavam comportamentos destrutivos significativos. $\mathrm{O}$ delineamento envolveu uma combinação de múltiplos elementos e linha de base múltipla entre os sujeitos, o que foi apropriado para o objetivo do estudo e permitiu uma comparação entre os dois procedimentos: NCR denso versus um esquema de NCR mais pobre ou esvanecido (Hagopian et al., 1994).

A análise funcional indicou que a atenção social, na forma de uma leve reprimenda, exercia função mantenedora para os comportamentos destrutivos (variável dependente). Durante a primeira fase do tratamento, foi utilizado um delineamento com tratamentos alternados entre os esquemas densos e mais espaçados de NCR. Após as comparações dos dois esquemas, o NCR foi implementado com um componente de esvanecimento, no qual, toda vez que a taxa de comportamento destrutivo diminuía até o critério, o esquema de NCR (atenção independente da resposta) na próxima etapa era esvanecido (idem, 1994).

Houve diversas mudanças nos procedimentos durante o estudo para atender às necessidades de participantes específicos, e para tornar o tratamento mais eficaz - característica importante de pesquisas em Análise do Comportamento Aplicada. Por exemplo, o processo de esvanecimento precisou ser mais gradual após um aumento no comportamento destrutivo para uma das participantes. Isso representou uma modificação dos procedimentos de esvanecimento usados originalmente por Vollmer et al., (1993), em que os autores definiram critérios para aumentar os intervalos, mas não para 
ajustá-los em caso de intensificação do comportamento. Treinos de generalização entre terapeutas, entre ambientes e com a mãe foram um passo importante no cumprimento dos objetivos do tratamento e representaram uma extensão dos estudos anteriores de Vollmer et al. (1993).

Os resultados deste estudo sugerem que um esquema denso de NCR é necessário no início do tratamento e que um esquema de reforçamento mais pobre pode ser eficaz se utilizado com esvanecimento sistemático. A análise dos esquemas denso e espaçado sugere que a saciação pode desempenhar um papel nos efeitos do NCR, pelo menos quando o reforço é apresentado em um esquema denso. Os autores afirmaram que o NCR pode alterar a operação estabelecedora do comportamento através da eliminação de um estado de privação que causaria o aumento na probabilidade da resposta (Hagopian et al., 1994). Porém, a saciação não explica a redução no comportamento de três dos quatro participantes que estavam sob esquemas mais espaçados. Uma possibilidade seria a de que os esquemas pobres não eliminaram, mas atenuaram as condições de privação, o que poderia justificar as diferentes taxas de resposta nas duas condições. Os autores atribuíram o aumento da eficácia do esquema mais pobre após o esvanecimento aos efeitos da extinção. Dessa forma, parece que ambos os componentes desempenharam um papel na eficácia dos resultados do NCR. Assim, uma importante questão levantada por este estudo é relacionada à densidade da distribuição dos estímulos potencialmente reforçadores que poderia promover reduções no comportamento problemático. Os autores ressaltaram que se fosse possível obter reduções do comportamento destrutivo com um esquema menos denso no começo do tratamento, então seriam necessários menos passos de esvanecimento, tornando o tratamento mais eficiente e mais fácil de implementar (idem, 1994).

Lalli, Casey e Kates (1997) estenderam a pesquisa anterior sobre NCR, conduzindo uma investigação dos parâmetros sob os quais o tratamento da NCR seria mais eficiente. O esquema de reforçamento no início do tratamento e a necessidade do uso de extinção associada ao procedimento de NCR foram explorados. Três crianças que estavam internadas em uma unidade hospitalar especializada em tratamento de comportamentos problemáticos severos participaram deste estudo. Na Fase 1, os resultados da análise funcional demonstraram que os comportamentos de hetero agressão da criança de 3 anos e autolesivos dos outros dois participantes eram mantidos pelo acesso a brinquedos preferidos por dois deles e uma atividade preferida (caminhada) por um dos participantes. Na Fase 2, foi implementado o NCR com extinção. A emissão de comportamentos problema não foi consequenciada e os reforçadores identificados durante a análise funcional como mantenedores dos comportamentos alvo foram entregues em um intervalo de tempo fixo (FT) que refletia a latência média para o primeiro comportamento problema de cada participante. O intervalo inicial para um dos participantes foi de 90 segundos e para os demais 120 segundos; esse intervalo foi sistematicamente ampliado até 600 e 720 segundos.

É importante notar que o esquema de distribuição temporal da apresentação de estímulos potencialmente reforçadores neste estudo difere das investigações anteriores, pois foi baseado em uma dimensão do comportamento do participante analisado durante a linha de base. Os resultados indicaram taxas mais baixas de comportamento-problema durante o NCR + extinção, em comparação com a linha de base, para os dois indivíduos que participaram dessa fase (Lalli et al., 1997).

Na Fase 3, o NCR foi implementado sem o componente de extinção para apenas um participante, a fim de determinar a necessidade do componente de extinção para reduzir os comportamentos-problema. Os procedimentos da Fase 3 foram semelhantes aos da Fase 2 deste estudo, exceto pelo fato de o terapeuta fornecer acesso aos objetos preferidos por 30 segundos contingente à ocorrência de comportamentos autolesivos e de acordo com intervalos de tempo fixos. Os resultados desta fase indicaram altos níveis de comportamentos autolesivos após a linha de base que reduziram nas sessões seguintes e níveis mais altos de comportamentos autolesivos na segunda linha de base em comparação com a primeira, mas os comportamentos autolesivos reduziram e não ocorreram após quatro sessões de reintrodução do tratamento (idem, 1997).

Os resultados deste estudo sugerem que não é necessário iniciar tratamento com reforçadores continuamente disponíveis. Estudos anteriores (Hagopian et al., 1994; Vollmer et al., 1993) mostraram que começar com esquemas contínuos 
ou densos foi eficaz para reduzir comportamentos problemáticos e também que seria preferível o início com um esquema denso ao invés de um esquema mais espaçado de reforço (Hagopian et al., 1994). Mas o uso de parâmetros derivados da linha de base, como a latência para a primeira resposta e o tempo entre as respostas, pode permitir a identificação de um intervalo que é rico o suficiente para alterar a operação estabelecedora para comportamentos problemáticos. É relevante mencionar que o uso de tais técnicas pode implicar, inicialmente, em etapas adicionais na aplicação do procedimento, uma vez que requer alguns cálculos, mas acabaria por significar menos sessões de esvanecimento e um processo de tratamento mais eficaz. Entretanto, os autores não compararam os esquemas contínuos com esquemas baseados em informações relativas à latência antes da primeira ocorrência e intervalo entre respostas; portanto, é necessária uma investigação aprofundada para garantir afirmações mais definitivas sobre os efeitos relativos dos esquemas. Além disso, o uso de NCR sem extinção foi testado com apenas um participante; os resultados precisariam ser replicados antes que uma conclusão precisa pudesse ser alcançada. A confirmação destes resultados indicaria que a saciação seria o componente do tratamento responsável pela redução de comportamento nos esquemas de NCR (Lalli et al., 1994).

Marcus e Vollmer (1996) examinaram mais sistematicamente os mecanismos responsáveis pelos efeitos do NCR e exploraram um possível efeito colateral do procedimento. Especificamente, eles investigaram se o esquema de reforço denso poderia levar à saciação, o que reduziria tanto a probabilidade de emissão do comportamento problema, quanto a motivação para exibir comportamentos alternativos. Para tanto, os autores sobrepuseram o esquema de DRA aos esquemas de NCR para determinar se mandos poderiam ser fortalecidos.

Três participantes com histórico de comportamentos autolesivos, heteroagressão e comportamentos disruptivos participaram deste estudo. A análise funcional demonstrou que os comportamentos-problema dos três participantes eram mantidos por acesso a itens tangíveis. Cada participante recebeu uma intervenção diferente a fim de se comparar os resultados do uso do NCR + DRA à sobreposição de DRA em um esquema previamente existente de NCR e aos efeitos de um esquema de DRA de intervalo fixo, no qual o reforço estava disponível em intervalos previamente especificados. No Experimento1, os pesquisadores implementaram um pacote com NCR e DRA, no qual os dois tratamentos foram administrados concomitantemente. Os resultados indicaram que o comportamento autolesivo do participante foi suprimido quase que imediatamente com a implementação de NCR e DRA, e as taxas de mando foram mantidas em níveis elevados durante o NCR contínuo; além disso, foi observado um aumento de mandos, à medida que o NCR era esvanecido. O fato de que o NCR não impediu o aumento na taxa dos mandos pode ser visto como uma indicação de que a supressão de respostas-alvo durante os procedimentos da NCR seria função da extinção (idem, 1996).

O Experimento 2 foi implementado para determinar os efeitos da sobreposição do procedimento de DRA a um esquema pré-existente de NCR. Dessa forma, os pesquisadores primeiramente liberaram o reforço apenas em um esquema de intervalo fixo (NCR) e na fase final incluíram o DRA. Os resultados deste experimento mostraram que quando o DRA foi adicionado no pacote de tratamento, primeiro notou-se um aumento dos comportamentos inadequados e depois estes reduziram para zero, enquanto os mandos foram estabelecidos e mantidos. Conforme mencionado pelos autores, uma progressão gradual dos tratamentos pode ser uma alternativa quando os cuidadores acham difícil implementar intervenções com múltiplos componentes.

No Experimento 3, Marcus e Vollmer (1996) combinaram o NCR e o DRA de tal maneira que as oportunidades de mandos por reforçadores estavam disponíveis apenas em momentos pré-estabelecidos (intervalo fixo de DRA); começando com um esquema denso e esvanecendo ao longo das sessões. De maneira congruente com os procedimentos de NCR, o reforçamento foi administrado independentemente da ocorrência de comportamentos problema. Os resultados indicaram que os comportamentos-alvo diminuíram durante os intervalos fixos de DRA; além disso, uma tendência descendente de mandos foi observada, sugerindo que o comportamento foram sensíveis às mudanças nos requisitos dos esquemas de intervalo fixo. 
Uma vantagem do uso do esquema de DRA com intervalos de tempo fixos em relação a outros esquemas diferenciais de reforçamento é que ele inclui a contingência de omissão (componente de extinção); mas ao mesmo tempo as ocorrências de comportamento problema, geralmente associadas com a ausência de reforço, são menos prováveis. Assim, o pacote de intervenção com NCR e DRA combinados mantêm as vantagens do NCR, enquanto aborda uma limitação importante: a ausência de contingências explícitas para aprender comportamentos adaptativos (idem, 1996).

Fisher, Iwata e Mazaleski (1997) ampliaram descobertas anteriores estudando a eficácia do uso de reforçadores arbitrários (não relevantes para a manutenção comportamental) como substitutos para os reforçadores funcionalmente equivalentes aos reforçadores que mantinham o comportamento problema (geralmente usados nas aplicações de NCR) e também abordando o papel da extinção na eficácia do NCR. Esses aspectos são muito relevantes, pois há determinadas situações em que não é possível disponibilizar o reforçador responsável pelo comportamento de forma não contingente; principalmente nos casos de comportamentos mantidos por reforçamento automático e outras ocasiões nas quais pais e cuidadores acabam respondendo aos comportamentos inadequados inadvertidamente ou porque o comportamento é muito severo e requer interrupção imediata.

Participaram do trabalho dois adultos (um homem e uma mulher) com déficit intelectual severo que moravam em uma instalação residencial para pessoas com transtornos no desenvolvimento. Ambos foram encaminhados para tratamento devido à apresentação de comportamentos autolesivos. A análise funcional demonstrou que tais comportamentos eram mantidos por reforçamento positivo. Foram selecionados três reforçadores arbitrários para cada participante, a partir de um conjunto de reforçadores em potencial, por meio de uma avaliação de preferências.

Fisher et al. (1997) usaram um delineamento de linha de base múltipla entre ambientes com um dos participantes e um delineamento com reversão para o outro, durante a fase em que o NCR foi testado. O reforçador arbitrário foi inicialmente entregue em um esquema de FT-10s, enquanto as ocorrências de comportamentos alvo continuavam a produzir o reforçador funcional (responsável pela manutenção do comportamento). Todavia, dada a probabilidade de um aumento na ocorrência de comportamentos autolesivos diante de um esquema pobre de NCR, antes de iniciarem o esvanecimento do esquema de reforçamento, primeiramente, introduziu-se o componente de extinção e somente quando a taxa de respostas por minuto diminuiu para $0.5 \mathrm{em}$ cinco sessões consecutivas é que foi iniciado o procedimento de esvanecimento, reduzindo-se a entrega do reforçador arbitrário de 6 para 5 por minuto e assim sucessivamente a cada sessão, desde que a taxa de autolesivos permanecesse menor que 0.5 por minuto durante a sessão prévia, até chegar-se a uma taxa de 0.2 por minuto.

Os resultados deste estudo indicaram que o acesso não-contingente a reforçadores arbitrários foi eficaz na redução de comportamentos autolesivos, mesmo quando continuavam a ser consequenciados com acesso a reforçadores. Dessa maneira, o estudo fornece suporte à perspectiva de que um esquema denso de reforçamento pode alterar as operações estabelecedoras dos comportamentos e afetar a frequência das respostas, mesmo quando o estímulo reforçador acessado não é funcionalmente equivalente àquele responsável pela manutenção do comportamento problema. Mas, é importante notar que, sob um esquema de reforçamento pobre, ou seja, mais espaçado, os autores consideraram plausível que os comportamentos autolesivos pudessem aumentar se continuassem sendo reforçados; portanto, o reforçamento não-contingente foi pareado com a extinção nesta fase. Assim, não está claro se a redução no comportamento associada ao NCR mais pobre foi devido a um efeito nas operações estabelecedoras dos comportamentos, ou em decorrência à extinção, ou em função da combinação dos dois. A necessidade de estudos mais aprofundados é sugerida, a fim de determinar qual seria o esquema de reforçamento mínimo necessário para produzir reduções significativas nos comportamentos autolesivos. Uma limitação importante no estudo de Fisher et al. (1997) foi a incapacidade de esvanecer o NCR para um dos participantes. Isto pode ser devido à relação entre os reforçadores arbitrários escolhidos para o estudo (itens comestíveis) e os comportamentos autolesivos do participante (morder a mão). 
Hanley, Piazza e Fisher (1997) investigaram a extensão com que estímulos alternativos altamente preferidos, identificados através de uma avaliação de preferência, poderiam reduzir comportamentos problemas mantidos por atenção, segundo análise funcional. Dois participantes do sexo masculino, de 11 e 16 anos, diagnosticados com déficit intelectual e admitidos para o tratamento de comportamentos agressivos, integraram o estudo. Foram criadas duas condições de tratamento, nas quais os participantes obtinham acesso à atenção ou a um item tangível preferido, que eram entregues de maneira nãocontingente em um esquema contínuo. Utilizou-se um delineamento misto, combinando tratamentos alternados e com reversão para dois os participantes.

Os resultados deste estudo indicaram que a apresentação não-contingente de atenção e a entrega não-contingente de itens tangíveis (altamente preferidos) foram eficientes em reduzir a frequência dos comportamentos destrutivos. Portanto, uma provável explicação é que os itens tangíveis substituíram eficazmente a atenção e alteraram a motivação dos participantes para exibir comportamentos agressivos (Hanley et al., 1997). Entretanto, diversas explicações alternativas também são plausíveis, entre elas a de que as reduções nos comportamentos alvos durante as fases do tratamento poderiam ser resultado da extinção, já que nenhuma consequência diferencial foi administrada para esses comportamentos. Nesse caso, a análise do NCR funcional ou arbitrário é pouco relevante. Também é possível que os itens tangíveis apresentados tivessem função de reforçadores; uma vez que essas variáveis não foram avaliadas diretamente. Diante do exposto, Hanley et al., (1997) sugerem que as conclusões devam ser consideradas com reservas.

É importante notar que os autores relatam não terem usado o esvanecimento nos seus estudos e não tentaram um esquema de reforçamento mais pobre, embora os autores tenham observado que os terapeutas precisam estar atentos ao risco de saciação como uma limitação potencial da substituição de estímulos preferidos alternativos pelo reforçador responsável pela manutenção do comportamento. Os comportamentos destrutivos mantidos por atenção poderiam aumentar se o indivíduo ficasse saciado desses estímulos substitutos.

Uma preocupação com o uso do NCR, que tem sido consistentemente levantada pelos profissionais da área (e.g., Fisher et al., 1997; Vollmer et al., 1993), diz respeito ao risco de reforçamento acidental do comportamento problemático durante a aplicação do procedimento em questão. Vollmer, Ringdahl, Roane e Marcus (1997) reportaram resultados de um procedimento de NCR que produziu explosões de extinção e reforçamento incidental.

Uma garota de 13 anos de idade com déficit intelectual severo, que tinha sido encaminhada para tratamento de agressividade, foi a participante do estudo de Vollmer et al. (1997). A análise funcional revelou que a agressão era diferencialmente sensível ao reforçamento positivo tangível. O tratamento consistiu na linha de base, seguida de NCR contínuo, depois uma reversão breve, e subsequentemente o NCR foi restabelecido com um componente de esvanecimento do esquema de reforçamento. Os efeitos colaterais negativos impediram o aumento do esquema além do intervalo fixo de 1 minuto. Foi adicionada uma condição final que incluía o reforçamento diferencial momentâneo de outros comportamentos (MDRO), no qual a entrega do reforço era interrompida se a agressão ocorresse cerca de 10 segundos antes da entrega programada.

Os resultados reportados por Vollmer et al. (1997) mostraram que o NCR pode promover explosões de respostas que podem culminar coincidentemente com a disponibilidade de reforço. As melhores práticas devem envolver uma análise cuidadosa dos padrões de resposta nas sessões e quando tais reforçamentos incidentais ocorrerem. Uma breve contingência de omissões, como o uso de MDRO, pode ser aconselhável para garantir resultados positivos. Outro procedimento que pode ser eficaz em reduzir o risco de reforçamento adventício é o uso de um esquema de reforçamento denso. Como observado por Fisher et al. (1997), é possível que, se houver apresentação de estímulo potencialmente reforçador não-contingente com densidade suficiente para reduzir consideravelmente a motivação, o fornecimento adventício do reforço não produza manutenção do comportamento. 
Todos os estudos mencionados envolveram o uso de reforço positivo não-contingente, porém a mesma lógica se aplica ao uso de reforçamento negativo não-contingente, como ilustrado por Vollmer, Marcus e Ringdahl (1995). Os autores examinaram o papel da fuga não-contingente (NCE) como um procedimento de extinção. Eles argumentaram que a NCE pode servir como extinção, uma vez que ela elimina a contingência entre os comportamentos auto-lesivos e a fuga e poderiam reduzir também as explosões de extinção, devido ao acesso não contingente aos reforçadores.

Dois jovens do sexo masculino, que foram encaminhados para o tratamento de comportamentos autolesivos crônicos, participaram do estudo. A análise funcional identificou que os comportamentos autolesivos eram mantidos por reforçamento negativo (fuga de demanda). Sendo assim, o reforçamento negativo não contingente (NCE) foi fornecido em um esquema de tempo fixo, independentemente da emissão de comportamentos autolesivos pelos participantes. Um participante também foi exposto ao reforçamento diferencial negativo de outros comportamentos (DNRO), no qual a fuga foi planejada, contingente à omissão dos comportamentos autolesivos por determinados períodos pré-estabelecidos (Vollmer et al., 1995). Os resultados deste estudo demonstraram que a NCE foi eficaz em reduzir os comportamentos autolesivos e produziu mudanças comparáveis às obtidas pelo DNRO. Além disso, o estudo mostrou que a extinção foi realizada utilizando intervalos de fuga que foram progressivamente aumentados. No entanto, a necessidade do componente de esvanecimento foi questionada pelos autores, que declararam que o componente principal da intervenção poderia ter sido apenas a extinção. Mas, uma vez que foi feita a implementação de todo um pacote de intervenção, não é possível determinar claramente quais foram os componentes ativos da intervenção.

\section{Meta Análises e Publicações Recentes}

Se os estudos da década de noventa já mostravam o quão promissor o procedimento de NCR poderia ser na redução de comportamentos problema, a vasta literatura produzida nos últimos vinte anos veio confirmar a efetividade do procedimento e o elevou ao rol de tecnologias baseadas em evidência. Pelo menos três revisões de literatura foram publicadas até hoje no JABA sobre NCR e cada uma delas concluiu que há evidências da efetividade do procedimento na redução de problemas de comportamento, e que determinados parâmetros devem ser observados para garantir a maior eficiência possível. Mas esses mesmos estudos, também apontam para a necessidade de pesquisas futuras, a fim de se obter uma melhor compreensão dos mecanismos que poderiam explicar não só os resultados favoráveis, mas inclusive algumas limitações evidenciadas pelas pesquisas realizadas até o momento.

Carr, Severtson e Lepper (2009) revisaram 59 aplicações de NCR funcional para problemas de comportamento mantidos por reforçamento social. Foram avaliados estudos nos quais se aplicou NCR com o uso de reforçadores funcionalmente equivalentes àqueles que foram identificados enquanto mantenedores do comportamento problema. Para tanto, foram excluídas as aplicações de NCR visando redução de comportamentos mantidos por reforçamento automático, haja vista que a condução deste tipo de procedimento nesses casos exige o uso de reforçadores alternativos ou estímulos 'competidores', que seriam aqueles itens associados a um alto grau de engajamento e interação concomitante à baixas taxas de comportamentos problema (Piazza et al., 1998). Essa limitação se deve ao fato de que muitas vezes não é possível se estabelecer definitivamente a função mantenedora quando as consequências são sensoriais, ou impedir o acesso ao reforçador. No máximo, pode-se utilizar o bloqueio da resposta com o objetivo de se prevenir reforçamento.

Dos 59 estudos incialmente selecionados por Carr e colaboradores (2009), 24 ofereceram suporte para o NCR funcional enquanto tratamento para problemas de comportamento de indivíduos com deficiência. Os autores utilizaram os critérios estabelecidos pela Força Tarefa da Divisão 12 da Associação Americana de Psicologia (1995), responsável pela promoção e divulgação de procedimentos psicológicos empiricamente embasados. Segundo os critérios estabelecidos pela Força Tarefa, um tratamento deve ser considerado como "bem estabelecido" quando existem evidências na literatura (pelo 
menos nove estudos) demonstrando, em pesquisas com delineamento experimental, que este é superior a outros tratamentos ou à ausência de tratamento. Ademais, pesquisadores devem utilizar procedimentos apresentados em manuais ou definir operacionalmente a variável independente e descrever claramente as características da sua amostra (população). A fim de quantificar o tamanho dos efeitos obtidos nas intervenções analisadas, foram utilizadas duas medidas: porcentagem de dados não sobrepostos (Nonoverlapping Data - PND) e redução da média dos valores da linha de base (Mean Baseline Reduction $M B R L)$. A PND foi determinada calculando a porcentagem de pontos no gráfico em uma determinada condição de tratamento abaixo do menor valor da linha de base; a MBLR foi calculada subtraindo a média dos valores na condição de tratamento da média dos valores da linha de base e então, dividindo essa diferença pela média da linha de base e multiplicando o quociente por 100 .

Os resultados da revisão de Carr et al (2009) indicaram que o procedimento de NCR com esvanecimento combinado ao uso de extinção pode ser considerado um procedimento "bem estabelecido". Onze estudos demonstraram a sua eficácia com vinte e cinco participantes. O valor médio do esquema de reforçamento inicial nesses estudos foi de FT 33.4 s (10-120 s) e o valor terminal médio foi de 311.7 s (60-720s.). O PND médio e os valores da MBLR para os tratamentos que utilizaram NCR com esvanecimento e extinção foram de $70.7(\mathrm{SD}=38.1)$ e 85.1 ( $\mathrm{SD}=16.2)$ respectivamente. As categorias de NCR sem extinção demonstraram um suporte empírico menor do que quando extinção era utilizada, porém esses dados devem ser considerados com cautela devido ao número reduzido de estudos nessa classificação.

Richman, Bernard-Brak, Grubb, Bosh e Abby (2015) realizaram uma meta-análise dos resultados de 55 estudos que utilizaram NCR na redução de problemas de comportamento. Os autores compararam os resultados dos estudos que utilizaram NCR com reforçadores funcionais com os que utilizaram reforçadores não funcionais, e documentaram os efeitos do esvanecimento do esquema de reforçamento nos resultados. Dados da meta-análise indicaram que NCR pode ser considerado um procedimento baseado em evidências e associado com uma redução significativa em problemas de comportamento. Ademais, constatou-se que o uso de um reforçador funcional aumenta a eficácia do procedimento se comparado ao uso de um reforçador não funcional, indicando que se deve realizar uma análise funcional quando houver possibilidade e utilizar reforçadores funcionalmente equivalentes aos que mantêm o comportamento problema. Não foi possível avaliar os efeitos do esvanecimento do esquema de apresentação dos estímulos (redução da densidade de apresentação de estímulos potencialmente reforçadores) devido ao pequeno número de artigos com a inclusão dessa manipulação que atingiram critério para participação no estudo. Foram identificados alguns episódios de aumento brusco em problemas de comportamento com redução nas sessões seguintes, durante diminuição da densidade de apresentação de estímulos potencialmente reforçadores, mas a análise estatística desses efeitos ficou prejudicada pelo número baixo de exemplares. Portanto a diminuição da densidade do esquema de reforçamento é algo que deve ser tratada com cautela e planejada de forma sistemática.

O esvanecimento no esquema de apresentação do estímulo potencialmente reforçador é comumente utilizado durante NCR para aumentar a aplicabilidade do procedimento em ambientes naturais. Por exemplo, não é nada prático e provavelmente insustentável solicitar que um pai ou uma mãe utilize NCR a cada 10 ou mesmo 30 segundos no decorrer do dia, pois certamente interferiria na execução de outras tarefas; mas aplicar o procedimento a cada 10 minutos já poderia ser mais razoável (Fisher et al., 1999). Outra razão para o esvanecimento se baseia na constatação de que o acesso livre e denso ao reforçador durante o procedimento de NCR tende a reduzir a motivação não só para o comportamento problema, como também para as respostas alternativas funcionalmente equivalentes. Ou seja, um esquema denso de NCR pode interferir, por exemplo, no treino de comunicação funcional FCT (Functional Communication Training), que envolve o ensino de respostas mais adequadas e com a mesma função da resposta que se deseja reduzir, pois afeta a operação motivadora para a emissão da resposta alternativa (Carr \& Durand, 1985; Tiger, Hanley, \& Brusek, 2008). 
Mas se o esvanecimento é necessário para facilitar a aquisição de novas respostas, também é verdade que há uma probabilidade de que as ocorrências de comportamento problema aumentem quando se inicia o esvanecimento; daí a importância de se combinar o uso de extinção aos procedimentos de NCR e FCT.

Phillips, Iannaccone, Rooker e Hagopian (2017) utilizaram um delineamento envolvendo uma série de casos consecutivos controlados para ampliar a literatura sobre NCR, onde avaliaram os resultados de 27 aplicações de NCR no tratamento de problemas de comportamento. Em 15 casos, NCR foi utilizado na redução de problemas de comportamento mantidos por reforçamento social; em nove casos NCR foi utilizado na redução de problemas de comportamento mantidos por reforçamento automático; em dois casos a análise funcional foi inconclusiva e em um caso, identificou-se reforçamento automático e social. Em 20 dos 27 casos, o NCR associado à extinção foi suficiente para obter-se uma redução de pelo menos $80 \%$ na ocorrência de problemas de comportamento; em sete casos houve necessidade da adição de outros componentes ao pacote de intervenção. Outro resultado importante desse estudo refere-se à observação de que NCR (não funcional) + bloqueio (prevenindo o acesso ao reforçador contingente á emissão do comportamento alvo) foi efetivo para menos de 50\% dos casos com problemas de comportamento mantidos por reforçamento automático inicialmente, sendo resultados mais satisfatórios obtidos somente quando NCR foi suplementado por componentes adicionais (como punição e FCT).

Os estudos apresentados somados aos resultados das meta-análises mais recentes demonstram que o papel do NCR combinado ao uso de extinção para reduzir comportamentos problemáticos mantidos por reforçadores sociais está bem estabelecido, e sua eficácia parece derivar de uma combinação de seus componentes. Todavia a efetividade do procedimento de NCR utilizando reforçadores não funcionais e sem ser combinado à extinção ainda precisa ser mais explorada, bem como questões relativas ao esvanecimento da densidade do reforçamento e à taxa terminal necessária para manutenção dos ganhos alcançados durante a intervenção.

Fritz, Jackson, Stiefler, Wimberly e Richardson (2017) avaliaram os efeitos do NCR sem extinção no tratamento de problemas de comportamento mantidos por reforçamento social positivo, combinado com a introdução de um esquema de reforçamento alternativo (FCT) durante a fase de esvanecimento do esquema de NCR. Participaram do estudo cinco crianças que frequentavam um centro de tratamento por pelo menos 15 horas semanais. Do grupo de participantes, quatro tinham diagnóstico de autismo e uma de transtorno obsessivo compulsivo. Os resultados de uma análise funcional (FA) indicaram "acesso a itens" como função mantenedora dos comportamentos de todos eles. Durante a intervenção, os mesmos itens altamente preferidos e utilizados durante a FA foram entregues em um esquema de NCR (todas as condições), contingente ao comportamento problema (todas as condições) e contingente ao mando (apenas na condição NCR + DRA).

Durante a fase de aplicação do NCR, os itens preferidos foram entregues por 20s em um esquema controlado por tempo e por 20s contingente à apresentação de comportamento problema. Para todos os participantes, a fase de NCR foi iniciada com três reforçamentos por minuto, o que implicava em acesso contínuo ao item preferido. O procedimento de esvanecimento foi idêntico ao utilizado por Marcus e Vollmer (1996) no qual o esquema de reforçamento foi reduzido para 2 por minuto, 1 por minuto e assim sucessivamente até chegar a 0.2 por minuto, sempre que os problemas de comportamento se mantivessem abaixo de $20 \%$ da taxa de linha de base. Se em qualquer momento durante o esvanecimento os problemas de comportamento fossem maiores que $20 \%$ dos valores de linha de base sem mostrar tendência decrescente em cinco sessões, era introduzido o treino de mando. NCR sem extinção foi efetivo em reduzir os comportamentos de três participantes. O esquema terminal de NCR com FT $5 \mathrm{~m}$ foi atingido em 30 sessões, aproximadamente. Dois participantes demostraram aumento na taxa de emissão dos comportamentos problema durante o procedimento de esvanecimento e necessitaram passar pela fase adicional de NCR + DRA.

Antes de iniciarem a condição de NCR + DRA foi feito um treino de mando utilizando encadeamento ao revés no qual os participantes aprenderam a entregar um cartão como forma de solicitação do item desejado. Uma vez iniciada a 
condição de NCR+ DRA, o reforçador passou a ser entregue em um esquema de tempo fixo, contingente ao problema de comportamento e mediante o pedido. Os participantes tiveram acesso contínuo aos reforçadores no começo dessa fase, e subsequentemente, iniciou-se o esvanecimento tal como feito anteriormente, porém nessa etapa o cartão para o mando estava disponível durante todo o período. Problemas de comportamento foram reduzidos para zero ocorrências nesse contexto.

Esses resultados ampliam aqueles obtidos nos estudos de Marcus e Vollmer (1996), demonstrando que a combinação de um esquema de FCT ao esquema de NCR durante a fase de esvanecimento pode aumentar a efetividade dos resultados, mesmo quando não se usa extinção. Todavia, a frequência de mandos emitidos pelos participantes foi bastante alta e relativamente próxima à taxa de problemas de comportamento apresentada durante a linha de base. Por conseguinte, ainda seria necessário reduzir o acesso contingente ao mando para que esse procedimento pudesse ter efeitos práticos mais significativos. Os autores sugerem que o uso de esquemas múltiplos de reforçamento poderia auxiliar no controle de estímulo sobre o mando, ou mesmo no estabelecimento de outros comportamentos contextualmente apropriados. Através da introdução gradual do atraso baseado em contingências, por exemplo, poder-se-ia reduzir a taxa de mandos e manter os comportamentos problema ausentes.

O uso de esquemas múltiplos de reforçamento pode facilitar um aumento na discriminação entre os componentes e aumentar a tolerância a períodos de extinção. Slocum, Grauerholtz-Ficher, Peters e Vollmer (2018) inicialmente implementaram NCR com três indivíduos com diagnostico de autismo e comportamentos agressivos mantidos por reforçamento social positivo (acesso a itens), usando um esquema denso de reforçamento. A densidade do esquema de reforçamento foi gradualmente reduzida incorporando múltiplos recursos com a finalidade de minimizar a probabilidade de ocorrência de problemas de comportamento durante o esvanecimento. Por exemplo, os pesquisadores incorporaram sinais indicando quando o reforçador estava ou não disponível e ofereceram acesso a potenciais reforçadores alternativos (atenção) quando o reforçador funcional era restrito. Foi possível reduzir o esquema de reforçamento em $50 \%$ ( 1 ou 2 por minuto) e manter os comportamentos problema em zero ocorrências em mais de $90 \%$ das sessões realizadas. Todavia, o esquema terminal desse estudo (1 a 2 reforçamentos por minuto) associado à atenção continuada, provavelmente não é sustentável de ser mantido com integridade pelos cuidadores no dia a dia. Novas análises dos componentes da intervenção podem ajudar a esclarecer quais condições são suficientes e necessárias para um esvanecimento do esquema de reforçamento com sucesso até uma densidade terminal possível de ser sustentada no ambiente natural.

Saini, Fisher e Pisman (2017) planejaram um arranjo analógico para avaliar e comparar os efeitos do uso de NCR +EXT como os efeitos do NCR sem EXT e verificar se a exposição prévia a esses arranjos afetaria a ressurgência das respostas quando fosse interrompida a entrega de reforçadores. Três crianças entre 5 e 6 anos de idade e com diagnóstico de autismo fizeram parte do estudo. Primeiramente os pesquisadores realizaram uma avalição de preferências e logo ensinaram os participantes a emitirem uma resposta simples de pressionar um botão ( 2 participantes) ou colocar uma ficha através de uma abertura em uma caixa (1 participante). Após essa fase de pré-treino, foi iniciada a linha de base durante a qual pressionar o botão ou depositar a ficha (respostas análogas ao comportamento problema) produzia reforçamento em um esquema de VI $30 \mathrm{~s}$ em ambas as condições. Na fase seguinte, os pesquisadores introduziram um esquema de reforçamento de VT $30 \mathrm{~s}$. Em uma condição experimental foi mantida o esquema de reforçamento da linha de base mais o novo esquema de VT 30s (NCR sem EXT), enquanto na outra condição de tratamento analógico, o esquema de reforçamento da linha de base foi interrompido $(\mathrm{NCR}+\mathrm{EXT})$. Por fim, na terceira fase, o reforçamento foi interrompido em ambas as condições (EXT). Para todos os participantes a ressurgência durante a terceira fase foi maior na condição associada com NCR sem extinção, na qual houve reforçamento continuado da resposta alvo e por conseguinte, maior taxa de reforçamento (VI+VT).

Segundo os autores, de acordo com a Teoria do Momentum Comportamental, mesmo quando NCR sem extinção produz uma redução significativa nos comportamentos, é provável que essa intervenção gere persistência e maior 
probabilidade de relapso. A Teoria do Momentum Comportamental propõe que, enquanto a taxa de respostas operantes depende das contingências entre resposta e reforçador, a resistência à extinção e/ou persistência da resposta é primariamente função da contingência entre o estímulo discriminativo e o reforçamento obtido na presença desse estímulo específico (Nevin \& Grace, 2000; Nevin, Mandel, \& Atek, 1983). Ou seja, contingências Pavlovianas de pareamento respondente (EstímuloReforçador) podem interferir na extinção do comportamento problema quando este é exposto a uma combinação de esquemas de reforçamento, como é o caso nas intervenções de NCR sem extinção, geralmente utilizadas no tratamento de comportamentos mantidos por reforçamento automático, nas quais um esquema de reforçamento baseado em intervalos de tempo (NCR) é introduzido concomitantemente a um esquema de reforçamento baseado na emissão de uma determinada resposta (geralmente em reforçamento contínuo). Tal fenômeno seria atribuído a um aumento no número de pareamentos entre estímulo e reforçador na condição NCR sem extinção. Como resultado dessa relação histórica, haveria uma maior probabilidade de ressurgência do comportamento, quando eventualmente se introduz uma condição de extinção. A consideração dessa limitação é de extrema importância quando se considera a manutenção dos efeitos clínicos de uma intervenção.

\section{Considerações Finais}

A partir dos estudos analisados, algumas qualificações são necessárias: primeiro, quando o NCR é implementado como a única intervenção e consequências para o comportamento problema permanecem inalteradas, sua influência nas operações motivadoras do comportamento parece depender da frequência da entrega ou disponibilização de itens e atividades preferidas. Em estudos futuros, uma análise precisa da latência até a emissão da primeira resposta e do intervalo entre respostas pode se instrumental na definição dos parâmetros mais eficientes de intervenção, sem explosões de extinção e com redução rápida do comportamento alvo. Segundo, quando o procedimento de NCR é implementado com extinção, sua eficácia sob esquemas pobres ou menos densos parece resultar da extinção e não da programação do NCR. Tampouco sabe-se ao certo o quão pobre ou espaçado pode ficar o esquema após o esvanecimento e ainda se manter os ganhos almejados. Tal como sugerido por Vollmer et al. (1995), uma análise do padrão de respostas de minuto a minuto, pode ser instrumental para a identificação dos parâmetros mais eficientes nas sessões de esvanecimento do NCR.

Pesquisas futuras também são necessárias para avaliar a influência de esquemas específicos sobre o comportamento problema a longo prazo. Não há dados suficientes informando o que ocorre com o comportamento problema quando a intervenção é finalizada. É possível que os efeitos sejam apenas transitórios, ou que haja necessidade de se manter a intervenção ativa por um maior período a fim de se obter resultados mais permanentes e generalizáveis no ambiente natural.

\section{Referencias}

Carr, E. G., \& Durand, V. M. (1985). Reducing behavior problems through functional communication training. Journal of Applied Behavior Analysis, 18(2), 111-126 10.1901/jaba.1985.18-111

Carr, E. G., \& Severtson, J. M. (2005). On the appropriateness of the term "noncontingent reinforcement". European Journal of Behavior Analysis, 6, 21-24.

Carr, J. E., Severtson, J. M., \& Lepper, T. L. (2009). Noncontingent reinforcement as an empirically supported treatment for problem Behavior exhibited by individuals with developmental disabilities. Research in Developmental Disabilities, 30, 4-57.

10.1016/j.ridd.2008.03.002.

Catania, A. C. (1998). Learning (4th ed.). Upper Saddle River, NJ: Prentice-Hall.

Catania, A. C. (2005). The nonmaintenance of behavior by noncontingent reinforcement. European Journal of Behavior Analysis 89(6):8994. $10.1080 / 15021149.2005 .11434253$

Cooper, J. O., Heron, T. E., \& Heward, W. L. (2019). Applied Behavior Analysis (3a ed). Pearson Education 
Fisher, S. M., Iwata, B.A., \& Mazaleski, J. L. (1997). Noncontingent delivery of arbitrary reinforcers as a treatment for self-injurious Behavior. Journal of Applied Behavior Analysis, 30, 239-249.

Fritz, J. N., Jackson, L. M., Stiefler, N. A., Wimberly, B. S., \& Richardson, A. R. (2017). Noncontingent reinforcement without extinction plus differential reinforcement of alternative behavior during treatment of problem behavior. Journal of applied behavior analysis, 50(3), 590-599. https://doi.org/10.1002/jaba.395

Hagopian, L. P., Fisher, W. W., \& Legacy, S. M. (1994). Schedule effects of noncontingent reinforcement on attention-maintained destructive behavior in identical quadruplets. Journal of Applied Behavior Analysis, 27, 317-325.

Hanley, G. P., Piazza, C. C., \& Fisher, W. W. (1997). Non-contingent presentation of attention and alternative stimuli in the treatment of attention-maintained destructive behavior. Journal of Applied Behavior Analysis, 30, 229-237.

Iwata, B. A., Dorsey, M. F., Slifer, K. J., Bauman, K. E., \& Richman, G. S. (1982). Toward a functional analysis of self-injury. Analysis \& Intervention Developmental Disabilities, 2(1), 3-20

Iwata, B. A., Dorsey, M. F., Slifer, K. J., Bauman, K. E., \& Richman, G. S. (1994). Toward a functional analysis of self-injury. Journal of Applied Behavior Analysis, 27, 197-209. 10.1901/jaba.1994.

Lakatos, E. M., \& Marconi, M. A. (2017). Fundamentos de Metodologia Científica. (7a ed.). Atlas.

Lalli, J. S., Casey, S. D., \& Kates, K. (1997). Noncontingent reinforcement as treatment for severe problem behavior: Some procedural variations. Journal of Applied Behavior Analysis, 30, 127-137.

Marcus, B. A., \& Vollmer, T. R. (1996). Combining non-contingent reinforcement and differential reinforcement schedules as treatment for aberrant behavior. Journal of Applied Behavior Analysis, 29, 43-51.

McSweeney, F. K., \& Norman, W. D. (1979). Defining behavioral contrast for multiple schedules. Journal of the Experimental Analysis of Behavior, 32(3), 457-461. 10.1901/jeab.1979.32-457.

Nevin JA, Mandell C, Atak JR. The analysis of behavioral momentum. Journal of the Experimental Analysis of Behavior. 1983; 39:49-59. DOI: 10.1901/jeab.1983.39-49 [PubMed: 16812312]

Nevin J.A., \& Grace R.C. (2000). Behavioral momentum and the law of effect. Behavior Brain Science 23(1):73-90. doi: 10.1017/s0140525x00002405. PMID: 11303339

Pereira, A. S., Sitsuka, D. M., Pereira, F. J., \& Shitsuka, R. (2018). Metodologia da Pesquisa Científica. Santa Maria, RS: UAB/NTE/UFSM.

Phillips, C. L., Iannaccone, J. A., Rooker, G. W., \& Hagopian, L. P. (2017). Noncontingent reinforcement for the treatment of severe problem behavior: An analysis of 27 consecutive applications. Journal of Applied Behavior Analysis, 50, 357-376. 10.1002/jaba.376.

Piazza, C. C., Fisher, W. W., Hanley, G. P., LeBlanc, L. A., Worsdell, A. S., Lindauer, S. E., \& Keeney, K. M. (1998). Treatment of pica through multiple analyses of its reinforcing functions. Journal of Applied Behavior Analysis, 31, 165-189.

Poling A., \& Normand M. P. (1999). Noncontingent Reinforcement: An Inappropriate Description of Time-Based Schedules That Reduce Behavior. Journal of Applied Behavior Analysis, 32, 237-238.

Reed, D. D., \& Kaplan, B. A. (2011). The matching law: A tutorial for practitioners. Behavior Analysis in Practice, 4(2), 15-24. 10.1007/BF03391780.

Richman, D. M., Barnard-Brak, L., Grubb, L., Bosch, A., \& Abby, L. (2015). Metaanalysis of noncontingent reinforcement effects on problem behavior. Journal of Applied Behavior Analysis, 48, 131-152. 10.1002/jaba.189.

Saini, V., Fisher, W. W., \& Pisman, M. D. (2017). Persistence during and resurgence following noncontingent reinforcement implemented with and without extinction. Journal of applied behavior analysis, 50(2), 377-392. https://doi.org/10.1002/jaba.380

Slocum, S. K., Grauerholz-Fisher, E., Peters, K. P., \& Vollmer, T. R. (2018). A multicomponent approach to thinning reinforcer delivery during noncontingent reinforcement schedules. Journal of applied behavior analysis, 51(1), 61-69. https://doi.org/10.1002/jaba.427

Tiger, J. H., Hanley, G. P., \& Bruzek, J. (2008). Functional communication training: A review and practical guide. Behavior Analysis in Practice, 1(1), 16-23. 10.1007/BF03391716.

Vollmer, T. R., Iwata, B. A., Zarcone, J. R., Smith, R. G., \& Mazaleski, J. L. (1993). The role of attention in the treatment of attention maintained selfinjurious behavior: Noncontingent reinforcement and differential reinforcement of other behavior. Journal of Applied Behavior Analysis, 26, 9-21.

Vollmer, T. R., Marcus, B. A., \& Ringdahal, J. E. (1995). Noncontingent escape as treatment for self-injurious behavior maintained by negative reinforcement. Journal of Applied Behavior Analysis, 28, 15-26.

Vollmer, T. R., Ringdahl, J. E., Roane, H. S., \& Marcus, B. A. (1997). Negative side effects of noncontingent reinforcement. Journal of Applied Behavior Analysis, 30, 161-164. 
Research, Society and Development, v. 10, n. 2, e33110212613, 2021

(CC BY 4.0) | ISSN 2525-3409 | DOI: http://dx.doi.org/10.33448/rsd-v10i2.12613

Vollmer, T. (1999). Noncontingent Reinforcement: Some Additional Comments. Journal of Applied Behavior Analysis, 32(2), 239-240. 